\title{
Anisotropic conformal infinity
}

\author{
Petr Hořava · Charles M. Melby-Thompson
}

Received: 10 September 2010 / Accepted: 23 October 2010 / Published online: 5 November 2010 (C) The Author(s) 2010. This article is published with open access at Springerlink.com

\begin{abstract}
We generalize Penrose's notion of conformal infinity of spacetime, to situations with anisotropic scaling. This is relevant not only for Lifshitz-type anisotropic gravity models, but also in standard general relativity and string theory, for spacetimes exhibiting a natural asymptotic anisotropy. Examples include the Lifshitz and Schrödinger spaces (proposed as AdS/CFT duals of nonrelativistic field theories), warped $A d S_{3}$, and the near-horizon extreme Kerr geometry. The anisotropic conformal boundary appears crucial for resolving puzzles of holographic renormalization in such spacetimes.
\end{abstract}

Keywords AdS/CFT correspondence $\cdot$ Holography for nonrelativistic field theories · Lifshitz and Schrödinger spacetimes · Conformal infinity of spacetime $\cdot$ Anisotropic scaling

\section{Contents}

1 Introduction . . . . . . . . . . . . . . . . . . . . . . . . . 1392

2 Anisotropic conformal infinity: the spatially isotropic case . . . . . . . . . . . . . . . . . . . 1392

2.1 The main idea . . . . . . . . . . . . . . . . . . . . . . . . . . . 1393

2.2 Asymptotic structure of the Lifshitz space . . . . . . . . . . . . . . . . . . . . . . . . . . 1394

3 Spatially anisotropic conformal infinity . . . . . . . . . . . . . . . . . . . . . . . . . . . . . . 1394

3.1 Asymptotic structure of null warped $A d S_{3} \ldots \ldots \ldots \ldots$. . . . . . . . . . . . . . 1395

3.2 Asymptotic structure of the Schrödinger space . . . . . . . . . . . . . . . . . . . . 1396

P. Hořava $(\bowtie) \cdot$ C. M. Melby-Thompson

Berkeley Center for Theoretical Physics, Department of Physics,

University of California, Berkeley, CA 94720-7300, USA

e-mail: horava@berkeley.edu

P. Hořava · C. M. Melby-Thompson

Theoretical Physics Group, Lawrence Berkeley National Laboratory,

Berkeley, CA 94720-8162, USA 
4 Holographic renormalization and anisotropic conformal infinity . . . . . . . . . . . . . . . . 1397

4.1 The general prescription . . . . . . . . . . . . . . . . . . . . . . . . . . . 1397

4.2 Asymptotic structure of spacelike warped $A d S_{3} \ldots \ldots \ldots$. . . . . . . . . . . . . . . . . 1398

5 Conclusions . . . . . . . . . . . . . . . . . . . . . . . 1399

\section{Introduction}

Recently, string theory and quantum gravity have begun to expand into territories traditionally associated with theoretical condensed matter physics. In the process, the apparent divide between relativistic and nonrelativistic systems is becoming significantly blurred. For example, relativistic gravity solutions have been proposed as duals of nonrelativistic quantum field theories (NRQFTs) [1,2] characterized by anisotropic scaling of time and space,

$$
t \rightarrow \lambda^{z} t, \quad x^{i} \rightarrow \lambda x^{i}
$$

with dynamical exponent $z \neq 1$. In another development, gravity models have been proposed [3-5] in which the gravitational field itself is subject to anisotropic scaling (1.1) at short spacetime distances, leading to an improved ultraviolet behavior.

At this new interface of condensed matter with quantum gravity, challenges and puzzles emerge. For example, extending the concept of holographic renormalization (see, e.g. [6] for a review) to nonrelativistic QFTs has proven surprisingly difficult. In standard holographic renormalization, the counterterms in a relativistic field theory are constructed from the analysis of the asymptotic behavior of bulk gravity near the boundary of spacetime. Many of the difficulties with holographic renormalization of NRQFTs can be traced to the fact that the proposed gravity duals have a degenerate conformal boundary, as defined in the sense of Penrose [7,8]. This degenerate behavior indicates that Penrose's definition of conformal infinity is insufficient to handle holography in such spacetimes, and that it needs to be generalized to incorporate systems with anisotropic scaling.

In this paper, we present such a generalization of conformal infinity of spacetime. Our construction is based on concepts first developed in the context of quantum gravity with anisotropic scaling $[3,4]$. Here we focus on the main idea of the construction, illustrated by a few examples; further details will appear elsewhere [9].

\section{Anisotropic conformal infinity: the spatially isotropic case}

One feature common to geometries dual to NRQFTs is that their asymptotic behavior "near the boundary" reflects the anisotropic scaling (1.1) of the dual NRQFT. This suggests that the correct notion of asymptopia and conformal infinity should reflect this anisotropy in the conformal transformations near the boundary. However, the idea of using anisotropic conformal transformations to define the boundary of spacetime immediately leads to apparent conflicts: the conformal boundary must be a geometric object, defined such that it is preserved by the symmetries of gravity; but spacetime 
diffeomorphisms only allow isotropic Weyl transformations, reducing us to Penrose's original definition.

\subsection{The main idea}

The observation crucial for resolving these conflicts was made [3] in gravity models with anisotropic scaling: Appropriately defined local anisotropic Weyl transformations are compatible with the restricted group $\operatorname{Diff}(M ; \mathcal{F})$ of those diffeomorphisms of spacetime $M$ that preserve a preferred foliation $\mathcal{F}$ of $M$ by fixed time slices. This fact allows us to define the concept of anisotropic conformal infinity, which legitimizes the asymptopia of many spacetimes, including those that appeared as duals of NRQFTs.

In the anisotropic gravity models of $[3,4]$, the reduction of symmetries to $\operatorname{Diff}(M ; \mathcal{F})$ is a consequence of the gauge symmetries of the system. However, our construction of anisotropic conformal infinity is valid beyond the context of [3,4], and applies naturally to a large class of solutions of standard general relativity and string theory: It is sufficient that the symmetries reduce to $\operatorname{Diff}(M ; \mathcal{F})$ only asymptotically, near the spacetime boundary. As we will see, this is indeed the behavior exhibited by the gravity duals of NRQFTs. This shows that the ideas of [3,4] find meaningful applications beyond the context of anisotropic gravity models.

The group $\operatorname{Diff}(M ; \mathcal{F})$ of foliation-preserving diffeomorphisms is generated by

$$
\xi \equiv f(t) \partial_{t}+\xi^{i}\left(t, x^{j}\right) \partial_{i}
$$

$\operatorname{Diff}(M ; \mathcal{F})$ appeared in $[3,4]$ as the gauge symmetry of gravity with anisotropic scaling (1.1). In the canonical (ADM) parametrization of the metric,

$$
d s^{2}=-N^{2} d t^{2}+g_{i j}\left(d x^{i}+N^{i} d t\right)\left(d x^{j}+N^{j} d t\right)
$$

the $\operatorname{Diff}(M ; \mathcal{F})$ generators act via

$$
\begin{aligned}
\delta_{\xi} N & =f \dot{N}+\dot{f} N+\xi^{i} \partial_{i} N, \\
\delta_{\xi} N_{i} & =f \dot{N}_{i}+\dot{f} N_{i}+\xi^{j} \partial_{j} N_{i}+\partial_{i} \xi^{j} N_{j}+\dot{\xi}^{j} g_{i j}, \\
\delta_{\xi} g_{i j} & =f \dot{g}_{i j}+\xi^{k} \partial_{k} g_{i j}+\partial_{i} \xi^{k} g_{j k}+\partial_{j} \xi^{k} g_{i k} .
\end{aligned}
$$

Using an arbitrary smooth nonzero scale factor $\Omega\left(t, x^{i}\right)$, we define the anisotropic Weyl transformations to be

$$
\widetilde{N}=\Omega^{z} N, \quad \widetilde{g}_{i j}=\Omega^{2} g_{i j}, \quad \widetilde{N}_{i}=\Omega^{2} N_{i}
$$

It was observed in $[3,4]$ that the generators $\delta_{\omega}$ of these anisotropic Weyl transformations form a closed algebra with the generators of $\operatorname{Diff}(M ; \mathcal{F})$ :

$$
\left[\delta_{\xi}, \delta_{\omega}\right]=\delta_{\varpi}, \quad \text { with } \quad \varpi=f \dot{\omega}+\xi^{i} \partial_{i} \omega
$$


Given (2.4), our definition of anisotropic conformal infinity of spacetime $M$ with metric $d s^{2}$ is essentially the same as in the isotropic case: We map $M$ by an anisotropic Weyl transformation $\Omega$ to an auxiliary spacetime $\widetilde{M}$ with a rescaled metric $\widetilde{d s^{2}}$, choosing $\Omega$ such that the region near infinity in $M$ is mapped to points inside a compact region of $\widetilde{M}$. Under this map, the ideal points at anisotropic conformal infinity of $M$ correspond to the boundary of the image of $M$ inside $\widetilde{M}$, where the scale factor $\Omega$ vanishes while $d \Omega \neq 0$. We will denote the anisotropic conformal infinity of $M$ by $\partial M$.

\subsection{Asymptotic structure of the Lifshitz space}

Our first example is the Lifshitz spacetime, with metric

$$
d s^{2}=-\frac{d t^{2}}{w^{2 z}}+\frac{d \mathbf{x}^{2}+d w^{2}}{w^{2}} .
$$

This geometry was proposed in [10] as the gravity dual for NRQFTs with Lifshitztype scaling without Galilean invariance. With the choice of $\Omega=w$, we find that the Lifshitz spacetime is anisotropically conformal to the portion of the flat spacetime with $w>0$, with the standard metric

$$
\widetilde{d s^{2}}=-d t^{2}+d \mathbf{x}^{2}+d w^{2} .
$$

The anisotropic conformal boundary at infinity in (2.6) is mapped to $w=0$ in (2.7). In this example, we see that

(a) the anisotropic boundary is of codimension one,

(b) the bulk metric induces an anisotropic conformal class of metrics in the boundary, and

(c) the action of the anisotropic conformal symmetry in the boundary is induced from the action of the bulk isometries.

Point (c) deserves a closer explanation: In analogy with the isotropic case, we define anisotropic conformal transformations of a fixed metric on $\partial M$ to be those $\operatorname{Diff}(\partial M ; \mathcal{F})$ transformations that map the metric to itself up to an anisotropic Weyl transformation. Here $-d t^{2}+d \mathbf{x}^{2}$ is a representative of the anisotropic conformal class of metrics at the boundary of the Lifshitz space. The corresponding group of anisotropic conformal transformations is finite-dimensional, generated by time translations, spatial translations and rotations, and the anisotropic scaling transformation (1.1). It is this conformal symmetry group whose action on $\partial M$ is induced from the bulk isometries of the Lifshitz space $M$.

\section{Spatially anisotropic conformal infinity}

Our other examples require a more refined structure, with several dynamical exponents and with nested foliations of spacetime. We consider the case with scaling

$$
t \rightarrow \lambda^{z} t, \quad x^{i} \rightarrow \lambda x^{i}, \quad y^{a} \rightarrow \lambda^{\zeta} y^{a},
$$


and look for anisotropic Weyl transformations which reduce for a constant $\Omega^{2} \equiv \lambda$ to (3.1), and form a closed group with those spacetime diffeomorphisms $\operatorname{Diff}\left(M ; \mathcal{F}_{2}\right)$ that preserve the structure of a nested foliation $\mathcal{F}_{2}$ of spacetime. $\operatorname{Diff}\left(M ; \mathcal{F}_{2}\right)$ is generated by

$$
\xi \equiv f(t) \partial_{t}+\xi^{i}\left(t, x^{j}\right) \partial_{i}+\eta^{a}\left(t, x^{j}, y^{b}\right) \partial_{a} .
$$

One could first assume that $g_{a b}$ is invertible, and simply iterate the logic from the single-foliation case. Examples with this behavior would include the obvious generalizations of the Lifshitz spacetime, with an additional spatial anisotropy and scaling (3.1); the anisotropic conformal infinity of such spacetimes again exhibits the same features (a)-(c) as in the single-foliation Lifshitz space.

We will be interested in a different class of examples, in which $g_{a b}$ is not necessarily invertible. The most interesting case corresponds to $\zeta=0$; spatial dimensions with this scaling will be called "ultralocal." We specialize to the case of just one ultralocal dimension $y$, and parametrize the metric as

$$
\begin{aligned}
d s^{2}= & g_{t t} d t^{2}+2 g_{t y} d t d y+g_{y y} d y^{2} \\
& +g_{i j}\left[d x^{i}+g^{i k}\left(A_{k} d t+B_{k} d y\right)\right]\left[d x^{j}+g^{j \ell}\left(A_{\ell} d t+B_{\ell} d y\right)\right] .
\end{aligned}
$$

Just as in the case of the single foliation $[3,4]$, the appropriate action of (3.2) on the fields of (3.3) can be obtained [9] by taking a nonrelativistic scaling limit of full spacetime diffeomorphisms Diff $(M)$, which results from substituting $A_{i} \rightarrow A_{i} / c, B_{i} \rightarrow$ $c B_{i}$, parametrizing the generators of $\operatorname{Diff}(M)$ as $\left(c f, \xi^{i}, \eta / c\right)$, and taking $c \rightarrow \infty$. This process yields transformation rules for the metric components under the action of $\operatorname{Diff}\left(M ; \mathcal{F}_{2}\right)$ which are compatible with the anisotropic Weyl transformations

$$
\begin{aligned}
g_{t t} & \rightarrow \Omega^{2 z} g_{t t}, g_{t y} \rightarrow \Omega^{2} g_{t y}, \quad g_{y y} \rightarrow g_{y y}, \\
g_{i j} & \rightarrow \Omega^{2} g_{i j}, A_{i} \rightarrow \Omega^{2} A_{i}, \quad B_{i} \rightarrow \Omega^{2} B_{i} .
\end{aligned}
$$

As we now illustrate in a number of examples, this version of anisotropic Weyl transformations again leads to a natural notion of anisotropic conformal infinity.

\subsection{Asymptotic structure of null warped $\mathrm{AdS}_{3}$}

Perhaps the simplest example is null warped $A d S_{3}$ [11],

$$
d s^{2}=-\frac{d t^{2}}{w^{4}}+\frac{2 d t d \theta+d w^{2}}{w^{2}} .
$$

We choose the global scaling of the coordinates to be

$$
t \rightarrow \lambda^{2} t, \quad w \rightarrow \lambda w, \quad \theta \rightarrow \theta
$$


This is an example of the scaling defined in (3.1). Using (3.4) with $\Omega=w$, the metric is mapped to

$$
\widetilde{d s^{2}}=-d t^{2}+2 d t d \theta+d w^{2}
$$

The anisotropic conformal boundary is again at $w=0$, and satisfies properties (a)-(c) just like the Lifshitz space, with one novelty: The group of anisotropic conformal symmetries - defined again as those $\operatorname{Diff}(\partial M ; \mathcal{F})$ elements that map the boundary metric $-d t^{2}+2 d t d \theta$ to itself up to an anisotropic Weyl transformation - is now infinite dimensional, with generators

$$
F(t) \partial_{t}+G(t) \partial_{\theta},
$$

with $F(t), G(t)$ arbitrary. Their action on the conformal class of metrics in $\partial M$ is induced by asymptotic $\operatorname{Diff}\left(M ; \mathcal{F}_{2}\right)$ isometries of null warped $A d S_{3}$. In the quantum theory (3.8) will give rise to a Virasoro algebra together with a $U(1)$ current algebra.

\subsection{Asymptotic structure of the Schrödinger space}

Our next example, the Schrödinger space

$$
d s^{2}=-\frac{d t^{2}}{w^{2 z}}+\frac{2 d t d \theta+d \mathbf{x}^{2}+d w^{2}}{w^{2}}
$$

has been proposed $[1,2]$ as a gravity dual of Galilean-invariant nonrelativistic CFTs with dynamical exponent $z$. In order to get a well-behaved anisotropic conformal infinity, we use the scalings of (3.1), with $x^{i} \equiv(w, \mathbf{x})$ and with $y \equiv \theta$ an ultralocal dimension. Using (3.4) together with $\Omega=w$ yields

$$
\widetilde{d s^{2}}=-d t^{2}+2 d t d \theta+d \mathbf{x}^{2}+d w^{2},
$$

with $\partial M$ again at $w=0$. Note an interesting feature: because $\theta$ scales with conformal exponent $\zeta=0$, this dimension is present both in the bulk and in the boundary, even if it is compactified; the conformal infinity is of codimension one. This interpretation of $\theta$ resolves some of the mysteries associated with this extra bulk dimension in holography of Schrödinger spaces.

The bulk isometries of (3.9) again induce the action of anisotropic conformal symmetries on the anisotropic conformal class $-d t^{2}+2 d t d \theta+d \mathbf{x}^{2}$ of boundary metrics. For example, in the case of $z=2$, this group of conformal transformations of $\partial M$ induced from the bulk isometries is generated by

$$
\begin{aligned}
& t^{2} \partial_{t}+t x^{i} \partial_{i}-\frac{1}{2} x^{2} \partial_{\theta}, \quad t \partial_{i}+x^{i} \partial_{\theta} \\
& t \partial_{t}+\frac{1}{2} x^{i} \partial_{i}, \quad \partial_{i}, \quad \partial_{\theta}, \quad x^{i} \partial_{j}-x^{j} \partial_{i} .
\end{aligned}
$$


These are the generators of the Schrödinger conformal group. Asymptotic bulk isometries formally extend this symmetry to an infinite-dimensional one $[9,12,13]$, analogous to (3.8).

The metric (3.9) describes the Schrödinger space in Poincaré-like coordinates. At least when $z=2$, it can be analytically continued beyond the Poincaré patch, to global Schrödinger space $[9,14]$

$$
d s^{2}=-\left(1+\frac{\hat{\mathbf{x}}^{2}}{\hat{w}^{2}}+\frac{1}{\hat{w}^{4}}\right) d \hat{t}^{2}+\frac{2 d \hat{t} d \hat{\theta}+d \hat{\mathbf{x}}^{2}+d \hat{w}^{2}}{\hat{w}^{2}}
$$

(3.9) and (3.12) are related by coordinate transformation

$$
\begin{aligned}
\hat{t} & =\arctan t, \hat{\theta}=\theta+\frac{t}{2\left(1+t^{2}\right)}\left(\mathbf{x}^{2}+w^{2}\right) \\
\hat{x}^{i} & =\frac{x^{i}}{\sqrt{1+t^{2}}}, \hat{w}=\frac{w}{\sqrt{1+t^{2}}} .
\end{aligned}
$$

It is reassuring that this transformation is a double-foliation preserving diffeomorphism, of the form (3.2). As a result, the anisotropic conformal boundary of global Schrödinger space can also be analyzed in our framework.

\section{Holographic renormalization and anisotropic conformal infinity}

In the few examples presented above, we simply determined the correct form of foliation-preserving diffeomorphisms and the correct anisotropic Weyl transformations by inspection. More complicated examples may involve multiple foliations and multiple anisotropies which obscure the precise details of the construction. It is therefore desirable to have an algorithmic tool for deriving the anisotropic asymptotic structure in more general cases.

We now outline how such rules can be systematically derived from considerations of holography in spacetimes with asymptotically anisotropic scaling.

\subsection{The general prescription}

The general prescription consists of the following steps:

(i) Identify consistent fall-off conditions on fields on $M$ (This step, which we take as an input, is a consequence of the precise definition of the dynamics, designed to identify a consistent phase space of the theory; see, e.g. [15-18]. for examples).

(ii) Identify the maximal subgroup of diffeomorphism symmetries compatible with (i).

(iii) Identify the anisotropic Weyl transformations compatible with (ii).

Given (i)-(iii), one can then relax the asymptotic fall-off conditions on the background to allow for a generic boundary metric $\gamma$, and derive the action of the asymptotic 
symmetries from (ii) on $\gamma$. This action yields the appropriately scaled version of appropriate foliation-preserving diffeomorphisms of $\partial M$ on the boundary metric $\gamma$. The anisotropic Weyl transformations on $\gamma$ are determined simply from the reparametrizations of the radial coordinate. Finally, we use (iii) to construct the anisotropic conformal infinity of $M$.

This general prescription can be illustrated with spacelike warped $A d S_{3}$ as an example.

\subsection{Asymptotic structure of spacelike warped $\mathrm{AdS}_{3}$}

The metric of the spacelike warped $A d S_{3}$ in global coordinates is [16]

$$
d s^{2}=-\left(1+r^{2}\right) d u^{2}+\frac{d r^{2}}{1+r^{2}}+\frac{4 v^{2}}{v^{2}+3}(r d u+d v)^{2} .
$$

Following steps (i)-(iii) outlined above, we get:

(i) Fall-off conditions on the deviations $h_{\mu \nu}$ of the metric from the background (4.1) were proposed in [18],

$$
\begin{aligned}
& h_{u u}=\mathcal{O}(r), \quad h_{v v}=\mathcal{O}\left(\frac{1}{r}\right), \quad h_{r r}=\mathcal{O}\left(\frac{1}{r^{3}}\right), \\
& h_{u v}=\mathcal{O}(1), \quad h_{r u}=\mathcal{O}\left(\frac{1}{r}\right), \quad h_{r v}=\mathcal{O}\left(\frac{1}{r^{2}}\right) \text {. }
\end{aligned}
$$

(ii) The group of diffeomorphisms preserving these fall-off conditions is generated by

$$
\left[F(u)+\mathcal{O}\left(\frac{1}{r^{2}}\right)\right] \partial_{u}-\left[r F^{\prime}(u)+\mathcal{O}\left(\frac{1}{r}\right)\right] \partial_{r}+\left[G(u)+\mathcal{O}\left(\frac{1}{r}\right)\right] \partial_{v},
$$

with $F(u), G(u)$ arbitrary, and exhibits a natural asymptotic foliation structure.

(iii) Given (4.3), we choose the anisotropic Weyl transformations

$$
g_{u u} \rightarrow \Omega^{4} g_{u u}, \quad g_{u v} \rightarrow \Omega^{2} g_{u v}, \quad g_{v v} \rightarrow g_{v v}
$$

on the metric. These are of the form (3.4), with $z=2$. Together with the asymptotic diffeomorphisms (4.3), the Weyl transformations form an algebra that closes up to subleading terms in $1 / r$. With the choice of $\Omega=r^{-1 / 2}$, we obtain the anisotropic conformal infinity of spacelike warped $A d S_{3}$. The boundary at anisotropic infinity is two-dimensional, and carries an induced anisotropic conformal class of metrics represented by

$$
-d u^{2}+\frac{4 v^{2}}{v^{2}+3}(d u+d v)^{2}
$$


The action of the correctly scaled form of $\operatorname{Diff}(\partial M ; \mathcal{F})$ on the boundary metric $\gamma(u, v)$ can be determined by relaxing the background to

$$
\begin{aligned}
g_{u u} & =r^{2} \gamma_{u u}(u, v)+\mathcal{O}(r), & g_{v v} & =\gamma_{v v}(u, v)+\mathcal{O}\left(\frac{1}{r}\right), \\
g_{u v} & =r \gamma_{u v}(u, v)+\mathcal{O}(1), & g_{r u} & =\mathcal{O}\left(\frac{1}{r}\right), \\
g_{r r} & =\frac{1}{r^{2}}+\mathcal{O}\left(\frac{1}{r^{3}}\right), & g_{r v} & =\mathcal{O}\left(\frac{1}{r^{2}}\right),
\end{aligned}
$$

and acting with the group of bulk diffeomorphisms which preserve this asymptotic form of the metric,

$$
\left[F(u)+\mathcal{O}\left(\frac{1}{r^{2}}\right)\right] \partial_{u}+\left[r H(u)+\mathcal{O}\left(\frac{1}{r}\right)\right] \partial_{r}+\left[G(u, v)+\mathcal{O}\left(\frac{1}{r}\right)\right] \partial_{v} .
$$

The radial bulk diffeomorphisms generated by $r H(u) \partial_{r}$ induce the correct anisotropic Weyl transformation (4.4) on the boundary metric, with $H$ as the generator. And the diffeomorphisms along $u$ and $v$ precisely reproduce the action of $\operatorname{Diff}(\partial M ; \mathcal{F})$ on $\gamma$ that we obtained from the $c \rightarrow \infty$ scaling below Eq. (3.3)! We can use this action of Diff $(\partial M ; \mathcal{F})$ to define the group of anisotropic conformal transformations of the boundary metric. This group is found to be generated by

$$
F(u) \partial_{u}+G(u) \partial_{v}
$$

For compact $u$, this reproduces the Virasoro algebra and the $U(1)$ current algebra found in [18]. A closely related example is the near-horizon extreme Kerr geometry $[17,19]$, which can be viewed as a family of spacelike warped $A d S_{3}$ 's, fibered over the polar coordinate $\theta$. In this example, $\theta$ is an ultralocal dimension, analogous to $\theta$ of the Schrödinger space (3.9), but without translational invariance along $\theta$.

\section{Conclusions}

The notion of anisotropic conformal infinity clarifies the asymptotic structure of vacuum spacetimes with asymptotically anisotropic scaling. As an application, we can now give a precise definition of black holes in spacetimes with anisotropic asymptopia: First, we define an event horizon in an asymptotically anisotropic spacetime as the boundary of the causal past of the anisotropic infinity, and define black holes as solutions with event horizons. Our definition of anisotropic conformal infinity naturally extends to the black holes themselves: For example, one can show that the spacelike warped $A d S_{3}$ black holes of [16] share the asymptotic structure of the spacelike warped $A d S_{3}$ vacuum determined above.

In relativistic gravity, the structure of conformal infinity is probed by null geodesics. Spacetimes with anisotropic scaling appearing in the context of $[3,4]$ can be similarly probed, by Lifshitz particles [9] with a gapless nonrelativistic dispersion relation. 
Results of this paper illustrate that under pressure from the interface of quantum gravity with condensed matter, some of the central notions of general relativity must be revisited and adapted for the era in which quantum gravity is applied to systems with anisotropic scaling. In the process, it appears that we must disentangle two concepts which seemed so inseparable in the physics of the twentieth century: gravitation and relativity.

Acknowledgments We wish to thank Stéphane Detournay for useful discussions. The results presented in this paper were announced by one of us (PH) at Strings 2009 in Rome (June 2009), and at the Quantum Criticality and AdS/CFT Correspondence Miniprogram at KITP, Santa Barbara (July 2009); PH wishes to thank the orgainzers for their hospitality. This work has been supported by NSF Grants PHY-0555662 and PHY-0855653, DOE Grant DE-AC02-05CH11231, and by the Berkeley Center for Theoretical Physics.

Open Access This article is distributed under the terms of the Creative Commons Attribution Noncommercial License which permits any noncommercial use, distribution, and reproduction in any medium, provided the original author(s) and source are credited.

\section{References}

1. Son, D.T.: Toward an AdS/Cold Atoms Correspondence: a geometric realization of the Schrödinger symmetry. Phys. Rev. D78, 046003 (2008). [arXiv:0804.3972]

2. Balasubramanian, K., McGreevy, J.: Gravity duals for non-relativistic CFTs. Phys. Rev. Lett. 101, 061601 (2008). [arXiv:0804.4053]

3. Hořava, P.: Membranes at quantum criticality. JHEP 03, 020 (2009). [arXiv:0812.4287]

4. Hořava, P.: Quantum gravity at a Lifshitz point. Phys. Rev. D79, 084008 (2009). [arXiv:0901.3775]

5. Hořava, P.: Spectral dimension of the universe in quantum gravity at a Lifshitz point. Phys. Rev. Lett. 102, 161301 (2009). [arXiv:0902.3657]

6. Skenderis, K.: Lecture notes on holographic renormalization. Class. Quant. Grav. 19, 5849-5876 (2002). [hep-th/0209067]

7. Geroch, R.P., Kronheimer, E.H., Penrose, R.: Ideal points in space-time. Proc. R. Soc. Lond. A 327, 545-567 (1972)

8. Penrose, R., Rindler, W.: Spinors and space-time, vol. 2. Cambridge University Press, Cambridge (1986)

9. Hořava, P., Melby-Thompson, C.M.: Anisotropic conformal infinity and the global structure of Schrödinger spaces (to appear)

10. Kachru, S., Liu, X., Mulligan, M.: Gravity duals of Lifshitz-like fixed points. Phys. Rev. D78, 106005 (2008). [arXiv:0808.1725]

11. Detournay, S., Orlando, D., Petropoulos, P.M., Spindel, P.: Three-dimensional black holes from deformed anti-de sitter. JHEP 07, 072 (2005). [hep-th/0504231]

12. Alishahiha, M., Fareghbal, R., Mosaffa, A.E., Rouhani, S.: Asymptotic symmetry of geometries with Schrödinger isometry. [arXiv:0902.3916]

13. Compère, G., de Buyl, S., Detournay, S., Yoshida, K.: Asymptotic symmetries of Schrödinger spacetimes. [arXiv:0908.1402]

14. Blau, M., Hartong, J., Rollier, B.: Geometry of Schrödinger space-times, global coordinates, and harmonic trapping. JHEP 07027 (2009). [ arXiv:0904.3304]

15. Brown, J.D., Henneaux, M.: Central charges in the canonical realization of asymptotic symmetries: an example from three-dimensional gravity. Commun. Math. Phys. 104, 207-226 (1986)

16. Anninos, D., Li, W., Padi, M., Song, W., Strominger, A.: Warped $A d S_{3}$ black holes. JHEP 03, 130 (2009). [arXiv:0807.3040]

17. Guica, M., Hartman, T., Song, W., Strominger, A.: The Kerr/CFT Correspondence. [arXiv:0809.4266]

18. Compère, G., Detournay, S.: Boundary conditions for spacelike and timelike warped $A d S_{3}$ spaces in topologically massive gravity. [arXiv:0906.1243]

19. Bardeen, J.M., Horowitz, G.T.: The extreme Kerr throat geometry: a vacuum analog of $A d S_{2} \times S^{2}$. Phys. Rev. D60104030 (1999). [hep-th/9905099] 